\title{
CO Mapping of Spiral Galaxies in the Ursa Major Cluster: An Atlas
}

\author{
Myung-Hyun Rhee \& Aeree Chung \\ Center for Space Astrophysics $\&$ Department of Astronomy, Yonsei \\ University, Seoul 120-749, Korea \\ Marc Verheijen \& Min S. Yun \\ NRAO, P.O. BOX 0, Socorro, NM 87801, USA
}

\begin{abstract}
We present the first results of the OTF CO $(\mathrm{J}=1-0)$ mapping of spiral galaxies in the Ursa Major cluster, obtained with the NRAO $12 \mathrm{~m}$ radio telescope during June 20-27, 1999 and March 23-30, 2000.
\end{abstract}

\section{Observations}

The properties of molecular gas in spiral galaxies is the subject of a wide field of research (e.g. Young \& Scoville 1991 and references therein) and much has been done on the global scale of galaxies. The advent and maturity of the On-The-Fly (OTF) mapping technique at the NRAO $12 \mathrm{~m}$ radio telescope now affords us with a way to address many issues on the more local scales within a large number of nearby spiral disks that are many arc minutes in diameter. We start a project which aims at OTF mapping of the distribution of $12 \mathrm{CO}(\mathrm{J}=1-0)$ emission in spiral galaxies in the Ursa Major cluster and compare in detail the distributions of the molecular gas to that of the neutral hydrogen as a function of the morphological type, surface brightness, dust content and temperature and the star formation threshold of a galaxy's disk.

We have obtained OTF CO ( $\mathrm{J}=1-0)$ maps with the NRAO $12 \mathrm{~m}$ telescope at Kitt Peak from two observational runs during June 20-27, 1999 and March 23-30, 2000. Among 23 galaxies observed, the CO emissions have been detected for 21 galaxies. Data reduction and analysis have been completed (Chung 2000). An example from the $\mathrm{CO}$ atlas is presented in Figure 1.

\section{References}

Chung, A. 2000, Masters' Thesis, Yonsei University

Young, J. S. \& Scoville, N. Z. 1991, ARA\&A 29, 581 


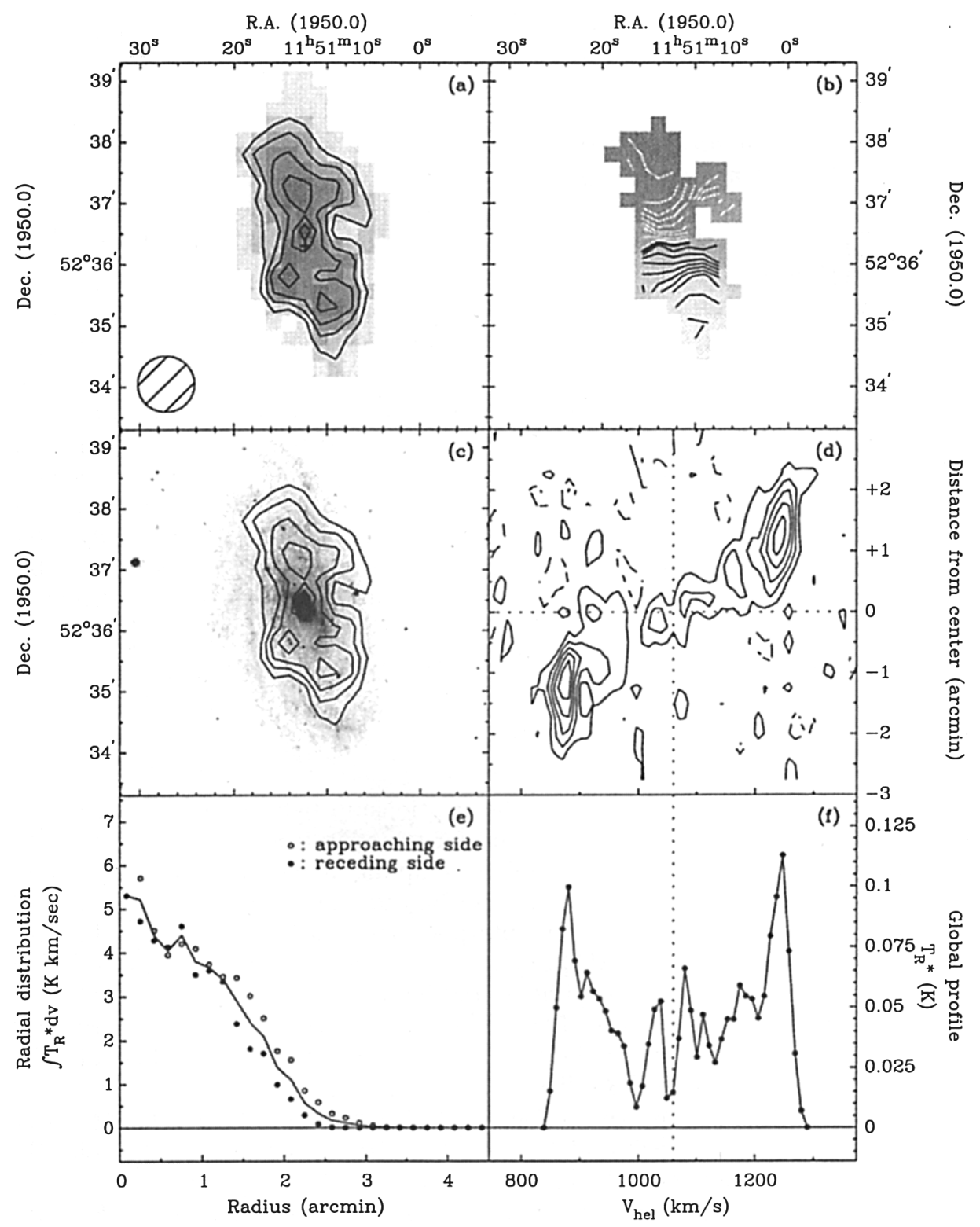

Figure 1. $\quad \mathrm{CO}$ atlas of NGC 3953. (a) Integrated CO map. (b) Velocity field diagram. (c) CO contours overlaid on B-band image. (d) Position-velocity diagram. (e) Radial distribution. (f) Global profile. 